\title{
Virtual Self-Training of a Sensory Substitution Device for Blind Individuals
}

\author{
Galit Buchs \\ Faculties of Medicine (IMRIC) \& \\ Cognition \\ Hebrew University of Jerusalem \\ Jerusalem, Israel \\ galit.buchs@mail.huji.ac.il
}

\author{
Benedetta Heimler \\ Faculties of Medicine (IMRIC) \& \\ Brain (ELSC) \\ Hebrew University of Jerusalem \\ Jerusalem, Israel \\ benedettaheimler@gmail.com
}

Liraz Braun

Faculties of Medicine (IMRIC)

Hebrew University of Jerusalem Jerusalem, Israel

liraz.braun@mail.huji.ac.il

\author{
Menachem Kerem \\ Faculties of Medicine (IMRIC) \\ Hebrew University of Jerusalem \\ Jerusalem, Israel \\ me.kerem@gmail.com
}

\author{
Shachar Maidenbaum \\ Faculty of Medicince (IMRIC), \\ Hebrew University of Jerusalem \\ Jerusalem, Israel \\ shachar.maidenbaum@mail.huji. \\ ac.il
}

\author{
Amir Amedi \\ Faculties of Medicine (IMRIC), \\ Brain (ELSC) \& Cognition \\ Hebrew University of Jerusalem \\ Jerusalem, Israel \\ amira@ekmd.huji.ac.il
}

\begin{abstract}
One of the main bottlenecks to the adoption of Sensory Substitution Devices (SSDs) in everyday life by blind users is the difficulty in learning to interpret their algorithms and the consequent dependency on sighted instructors throughout the learning-process. Here we test the efficacy of a virtual online selftraining program we developed for learning the basics of the EyeMusic, a visual-to-auditory SSD. Furthermore, to better understand the properties of self-training we tested the intuitiveness of the device, based on performance after a brief explanation but no exposure, and tested several variations on feedback during self-training.

We tested the performance of two groups of sighted participants via pre-post identical exams, with intermediate training lessons. These groups were offered different feedback after experiencing the auditory stimuli - either a visual version of the stimuli or a textual description.

After a brief explanation of the EyeMusic algorithm, and before training, participants scored $41 \pm 10.6 \%$ in the exam, significantly above chance. Self-training led to a highly significant improvement with a $59 \pm 12 \%$ score in the post-exam. No significant difference was found between the post-exam results of the two different feedback groups.

These results demonstrate the possibility to self-train on the basics of a whole-scene visual-to-auditory SSD. Furthermore, visual access to the images during training did not seem to improve the final score, demonstrating the potential efficacy of such self-training method also for blind users.
\end{abstract}

Keywords- sensory substitution, vision rehabilitation, multisensory processing, online training, self-training, visual impairment, blindness

\section{INTRODUCTION}

Offering visual information to the millions of blind individuals worldwide is a major rehabilitation goal [1]. There are continuous efforts searching for effective ways to provide blind people with information which is regularly conveyed through vision and thus is out of their reach. One promising set of tools in this prolific domain are visual-to-auditory Sensory Substitution Devices (SSDs). Visual-to-auditory SSDs are a family of non-invasive devices that convert visual input to audition according to a specific algorithm [2-3]. SSDs can potentially aid blind individuals in many situations. For example they have recently been shown successful for navigation and obstacle detection and avoidance as well as for various object recognition tasks with different degree of difficulty [4-5]. Yet, despite these impressive results under both lab conditions [4, 6] and in the real world [5], SSDs have not been widely adopted by the blind community outside the lab settings. Previous suggestions have implicated the lack of availability, cost and cumbersomeness of the setups [6]. These issues have been mitigated to a large extent by the rise in availability of smartphones enabling mobile compact and relatively cheap processing and sensing units. Thus at the moment, SSDs such as the vOICe and EyeMusic, are freely available (https://goo.gl/hpRW3R, https://goo.gl/NQlOCX) and do not require additional hardware beyond headphones.

The main issue currently highlighted as the bottleneck to SSDs wide adoption by the blind community is training [4, 7] as advanced use of these devices requires intensive training [8]. Training is indeed key to facilitate the interpretation of the SSD algorithm, ultimately making SSD use easier and less cognitively demanding.

One limitation with current SSD training programs is that they require the constant presence of a sighted trainer who will teach the trainee (blind/ sighted/ the researchers themselves) how to use the SSDs and interpret the information it provides.

In the attempt of loosening this bottleneck, we developed a new training tool: a self-training online program for learning the EyeMusic, a visual-to-auditory SSD developed in our lab. 
Here, we tested the efficacy of the initial stages of our selftraining program. As a secondary goal, we compared two multisensory training feedback methods within it, a "visual" and a "reading" approach (see methods for more details). The reading self-training approach was chosen since it can also be implemented for SSD blind users via text-readers.

Self-training can potentially facilitate the training program of visual-to-auditory SSDs, by replacing or complementing the one-on-one training, thus, promoting the overall use of SSDs.

\section{Methods}

\section{A. Equipment}

Visual input was translated into audio stimuli with the EyeMusic algorithm [8]. X-axis information was conveyed via a left-to-right sweep-line while height was preserved through pitch, i.e. high-pitched musical notes represent high locations in the image. Different musical instruments are used to convey different colors.

To maximize the usability and distribution of the selftraining program, we created an online version of the EyeMusic (http://brain.huji.ac.il/launch). One main advantage of an online version is that users do not need to install any program to train on the EyeMusic and can familiarize themselves with the algorithm at their own pace.

\section{B. Participants}

Nineteen sighted individuals, aged $26 \pm 13.4$ years (mean \pm SD), participated in this study. All participants were naïve to the EyeMusic algorithm. This experiment was conducted in accordance with the Helsinki declaration and all participants signed their informed consent.

\section{Experimental design}

We developed a self-training program for the EyeMusic SSD (http://brain.huji.ac.il/launch/Home/create_user_short) and tested its efficacy using a pre-post exam paradigm with a series of 9 step-by-step lessons in between ( $\sim 90$ minutes).

The pre-post exams were identical, including 30 4-AFC (Alternate Forced Choice) questions. The intermediate training lessons were of increasing difficulty, each followed by a midterm exam, with a $70 \%$ cut-off level (over this level participants moved on to the next lesson, otherwise they repeated the current lesson).

Participants were divided into two groups, "visual" or "reading", according to the type of feedback provided during training. In the "visual" group, the audio representation was followed by a visual image of the exact same stimulus, while the "reading" group read a textual description of it.

\section{RESUlTS}

After just a brief and generic explanation of the EyeMusic algorithm and before training, all participants in both groups answered correctly $41 \pm 10.6 \%$ of the questions in the pre-exam.
This success rate was significantly higher than the $25 \%$ chance level ( $\mathrm{P}<0.001$, Wilcoxon rank-sum). EyeMusic training led to a highly significant improvement $(\mathrm{P}<0.001$, Wilcoxon signed-rank) with a $59 \pm 12 \%$ success rate in the post-exam.

No significant difference was found between the two training feedback methods in the post-exam scores $(p=1$, Wilcoxon rank-sum). The "visual" group correctly answered $59 \pm 13 \%$ of questions, and the "reading" group correctly answered $59 \pm 11.5 \%$ of questions.

\section{DISCUSSION}

Our findings support the feasibility of self-training to learn the basics of the EyeMusic SSD algorithm, and suggests the potential benefit of adopting this strategy also for other SSDs. Crucially, our findings point to a simple way that can be further developed and might succeed in loosening the training bottleneck and in spreading the use of SSDs among blind individuals.

We found no significant additional benefit of either of the training feedback methods. Thus, these results suggest that self-training on the basics of our EyeMusic SSD can potentially be extended to blind participants as well (who can rely on textual description only, albeit conveyed auditory through voice-readers). Thus, our next stage will be testing this system, which has been already designed bearing in mind accessibility, with blind participants.

\section{ACKNOWLEDGMENT}

This work was supported by a European Research Council Starting-Grant (310809), a James S. McDonnel Foundation scholar award (no. 652 220020284), European Research Council Consolidator-Grant (773121).

\section{REFERENCES}

[1] World Health Organization, "Visual impairment and blindness-key facts and world demographics," 2018.

[2] S. Abboud, S. Hanassy, S. Levy-Tzedek, S. Maidenbaum, and A. Amedi, "EyeMusic: Introducing a "visual' colorful experience for the blind using auditory sensory substitution," Restor. Neurol. Neurosci., vol. 32, no. 2, pp. 247-257, 2014.

[3] P. B. L. Meijer, "An Experimental System for Auditory Image Representations," IEEE Trans. Biomed. Eng., vol. 39, no. 2, pp. 112121, 1992.

[4] S. Maidenbaum, S. Abboud, and A. Amedi, "Sensory substitution: Closing the gap between basic research and widespread practical visual rehabilitation," Neurosci. Biobehav. Rev., vol. 41, pp. 3-15, 2014.

[5] J. Ward and P. Meijer, "Visual experiences in the blind induced by an auditory sensory substitution device," Conscious. Cogn., vol. 19, no. 1, pp. 492-500, 2010

[6] G. V Elli, S. Benetti, and O. Collignon, "Is there a future for sensory substitution outside academic laboratories?," Multisens. Res., vol. 27, no. 5-6, pp. 271-291, 2014.

[7] S. Maidenbaum, R. Arbel, G. Buchs, S. Shapira, and A. Amedi, "Vision through other senses: practical use of Sensory Substitution devices as assistive technology for visual rehabilitation," in Control and Automation (MED), 2014 22nd Mediterranean Conference of, 2014, pp. 182-187.

[8] M. Auvray, S. Hanneton, and J. K. O'Regan, "Learning to perceive with a visuo - auditory substitution system: localisation and object recognition with 'The Voice,"” Perception, vol. 36, no. 3, pp. 416-430, 2007. 